\title{
RR Lyrae stars in the southern globular cluster NGC $362^{\star, \star \star}$
}

\author{
P. Székely ${ }^{1,2}$, L. L. Kiss ${ }^{3}$, R. Jackson ${ }^{3}$, A. Derekas ${ }^{3}$, B. Csák ${ }^{4}$, and K. Szatmáry ${ }^{1}$ \\ 1 Department of Experimental Physics \& Astronomical Observatory, University of Szeged, 6720 Szeged, Dóm tér 9., Hungary \\ e-mail: pierre@physx.u-szeged.hu \\ 2 Hungarian Eötvös Fellowship, School of Physics, University of Sydney, NSW 2006, Australia \\ 3 School of Physics, University of Sydney 2006, NSW 2006, Australia \\ 4 Department of Optics and Quantum Electronics, University of Szeged, 6720 Szeged, Dóm tér 9., Hungary
}

Received 13 August 2006 / Accepted 14 November 2006

\section{ABSTRACT}

\begin{abstract}
Aims. NGC 362 is a bright southern globular cluster in the foreground of the Small Magellanic Cloud (SMC), for which no extensive variability survey has ever been done. Finding regularly pulsating RR Lyrae stars in the cluster can lead to improved metallicity and distance estimates of the system, while other types of variable objects may be used to confirm the results.

Methods. Time-series CCD photometric observations have been obtained. Light curves have been derived with both profile fitting photometry and image subtraction. We developed a simple method to convert flux phase curves to magnitudes, which allows the use of empirical light curve shape vs. physical parameters calibrations. Periods and light curve parameters of the detected variable stars have been determined with Fourier analysis, phase dispersion minimization and string-length minimization. Using the RR Lyrae metallicity and luminosity calibrations, we have determined the relative iron abundances and absolute magnitudes of the stars. The color-magnitude diagram has been fitted with Yale-Yonsei isochrones to determine reddening and distance independently. For five RR Lyrae stars we obtained radial velocity measurements from optical spectra.

Results. We found 45 RR Lyr stars, of which the majority are new discoveries. While most of them are cluster members, as shown by their radial velocities and positions in the color-magnitude diagram, we also see a few stars in the galactic field and in the outskirts of the SMC. About half of the RR Lyraes exhibit light curve changes (Blazhko effect). The RR Lyrae-based metallicity of the cluster is $[\mathrm{Fe} / \mathrm{H}]=-1.16 \pm 0.25$, the mean absolute magnitude of the RR Lyrae stars is $M_{\mathrm{V}}=0.82 \pm 0.04$ mag implying a distance of $7.9 \pm 0.6 \mathrm{kpc}$. The mean period of RRab stars is $0.585 \pm 0.081$ days. These properties place NGC 362 among the Oosterhoff type I globular clusters. The isochrone fit implies a slightly larger distance of $9.2 \pm 0.5 \mathrm{kpc}$ and an age of $11 \pm 1 \mathrm{Gyr}$. We also found 11 eclipsing binaries, 14 pulsating stars of other types, including classical Cepheids in the SMC and 15 variable stars with no firm classification.

Conclusions. NGC 362 hosts a large number of RR Lyrae stars, which makes the cluster a potentially important test object for studying the Blazhko effect in a chemically homogeneous environment.
\end{abstract}

Key words. stars: variables: general - stars: variables: RR Lyr - globular clusters: individual: NGC 362

\section{Introduction}

Although globular clusters (GCs) play an important role in testing stellar evolutionary models, one can still find suprisingly bright neglected GCs, especially in the southern hemisphere. NGC 362 is such a cluster, having been target of relatively few studies and so far lacking any comprehensive search for variable stars, particularly in the central regions of the cluster. Apart from the pioneering work of Sawyer (1931), who presented data for fourteen variable stars, recent works mainly aimed at obtaining deep color-magnitude diagrams (CMDs, e.g. Alcaino 1976; Harris 1982; Bolte 1986, 1987; Bellazzini et al. 2001). In the catalog of globular cluster variables (Clement et al. 2001, Clement 2002) there are only 16 stars from NGC 362 and many are far from the cluster center and have ambiguous properties.

\footnotetext{
* Section 7 is only available in electronic form at http: //www . aanda. org

$\star \star$ Photometric data is only available in electronic form at the CDS via anonymous ftp to cdsarc.u-strasbg.fr (130.79.128.5) or via http://cdsweb.u-strasbg.fr/cgi-bin/qcat?]/A+A/463/589
}

We have been carrying out a CCD photometric survey of southern GCs since mid-2003. In this paper we discuss the results for NGC 362, which is located in front of the outer edge of the Small Magellanic Cloud (SMC). NGC 362 lies at the position $\alpha=01^{\mathrm{h}} 03^{\mathrm{m}} 14^{\mathrm{s}}, \delta=-70^{\circ} 50^{\prime} 53^{\prime \prime}(\mathrm{J} 2000.0), l=301^{\circ} 5$, $b=-46^{\circ} 2$, located at $8.5 \mathrm{kpc}$ from the Sun and $\sim 9.4 \mathrm{kpc}$ from the galactic center; the metallicity $([\mathrm{Fe} / \mathrm{H}])$ is around -1.16 , while the Horizontal Branch is at $V_{\mathrm{HB}}=15.44$. The reddening is almost negligible, $E(B-V)=0.05$ (Harris 1996).

Models for globular clusters suggest that all the member stars were formed from the same gas cloud at approximately the same time. Physical parameters of member stars should therefore be representative of the cluster itself. Recently, many empirical relations have been calibrated for RR Lyrae stars (Jurcsik \& Kovács 1996; Kovács \& Jurcsik 1996, Jurcsik 1998, Kovács \& Walker 2001), which can be used to determine metallicities, absolute magnitudes, reddenings and other physical parameters from the light curve shapes of these variables. Here we present an analysis of RR Lyrae stars in NGC 362 and briefly discuss properties of variable stars of other types. 
Table 1. Log of time series observations.

\begin{tabular}{lccc}
\hline \hline Date & Filter & Data points & Length [h] \\
\hline 2003 July 29 & $V$ & 15 & 1.20 \\
2003 August 2 & $V$ & 65 & 4.33 \\
2003 August 3 & $V$ & 42 & 2.80 \\
2003 August 4 & $V$ & 57 & 3.80 \\
2003 August 5 & $V$ & 42 & 2.80 \\
2003 August 7 & $V$ & 56 & 3.73 \\
2003 August 8 & $V$ & 25 & 1.67 \\
2003 August 9 & $V$ & 32 & 2.13 \\
2003 October 8 & $V$ & 31 & 2.06 \\
2003 October 10 & $V$ & 17 & 1.13 \\
2003 October 13 & $V$ & 52 & 3.46 \\
2003 October 14 & $V$ & 59 & 3.93 \\
2003 October 16 & $V$ & 64 & 4.26 \\
2003 October 17 & $V$ & 20 & 1.33 \\
2003 October 18 & $V$ & 19 & 1.26 \\
2004 October 2 & $V$ & 115 & 6.85 \\
2004 October 5 & $V$ & 106 & 7.46 \\
\hline
\end{tabular}

Landolt fields in $V$ and $I: 2003$ August 1

Table 2. Log of spectroscopic observations.

\begin{tabular}{lcc}
\hline \hline Date & No. of spectra & Stars \\
\hline 2004 October 25 & $9,1,10$ & V7, V27, V74 \\
2004 October 26 & $1,2,1$ & V7, V23, V74 \\
2004 October 27 & 1 & V7 \\
2004 October 28 & 17 & V7 \\
2004 October 29 & 12,9 & V23, V71 \\
2004 October 30 & 7 & V27 \\
2004 November 1 & 6,10 & V27, V74 \\
2004 November 2 & 1,4 & V7, V27 \\
\hline
\end{tabular}

\section{Observations and data analysis}

Time-series $V$-band CCD photometric observations were carried out on 17 nights between July 2003 and October 2004, using the $1 \mathrm{~m}$ ANU telescope at Siding Spring Observatory, Australia. For imaging we used three chips of the Wide Field Imager giving $\sim 40^{\prime} \times 26^{\prime}$ field of view. In addition to the photometry, we measured radial velocities for 5 RR Lyrae stars from optical spectra taken with the $2.3 \mathrm{~m}$ ANU telescope and the Double-Beam Spectrograph. We used only the red arm of the spectrograph with the $1200 \mathrm{~mm}^{-1}$ grating, covering the wavelength range 5800-6800 A. The nominal resolving power was $\lambda / \Delta \lambda \approx 7000$. With 20-min exposures, the spectra had $S / N$ ratios of $20-50$, depending on the weather conditions. The full journals of observations are summarized in Tables 1 and 2.

All data were processed with standard IRAF $^{1}$ routines, including bias and flat corrections. Cluster images were then analysed both with PSF photometry, using the daophot package in IRAF, and image subtraction photometry, using the ISIS 2.1 package of Alard (2000). For standard photometric calibrations we observed selected equatorial fields of Landolt (1992): SA 109-954, SA 110-503, SA 111-1965, Mark A and SA 113-241. The transformation coefficients were determined with the task

${ }^{1}$ IRAF is distributed by the National Optical Astronomy Observatories, which are operated by the Association of Universities for Research in Astronomy, Inc., under cooperative agreement with the National Science Foundation. photcal. Radial velocities were measured with cross-correlation, using spectra of the IAU radial velocity standard HD 187691 and the task fxcor, leading to an estimated accuracy of $\pm 7 \mathrm{~km} \mathrm{~s}^{-1}$.

Light curves from PSF-fitting and image subtraction were analysed for finding periods. For this, we applied a combination of Fourier analysis, phase dispersion minimization (Stellingwerf 1978) and string-length minimization (Lafler \& Kinman 1965). The latter gives better results when the light curve contains sharp features, such as narrow minima of Algol-type eclipsing binaries. Every light curve was then inspected visually and classified according to its period, amplitude and shape. Variable star designations were assigned in an increasing order of right ascension.

Image subtraction is far more efficient than PSF photometry in detecting variable stars in crowded fields. Globular cluster studies are heavily affected by this, where large incompleteness factors can arise from the highly concentrated distributions of stars (Kaluzny et al. 2001). Even for the best-studied clusters, image subtraction can yield new discoveries (e.g. Clementini et al. 2004). There is, however, a major practical difficulty in using image subtraction results for the empirical light curve shape vs. physical parameters calibrations. Whereas ISIS gives time variations of brightness expressed in differential fluxes (relative to a master CCD frame), all the empirical relations were calibrated with light curves in magnitudes, which have a different shape, especially for the largest-amplitude objects. To convert fluxes to magnitudes, one has to determine a reliable magnitude estimate for every variable star in the master CCD frame, otherwise the conversion (and employing the calibrations) is not possible (see, e.g., Corwin et al. 2006).

Since we detected about twice as many RR Lyrae stars with ISIS 2.1 than with daophot, we developed a simple method for a flux-to-magnitude conversion. The main assumption is that there is a unique scaling between the mean-subtracted ISIS fluxes and flux values calculated from the mean-subtracted daophot magnitudes, so that one can write the following equation:

$$
10^{-0.4(m-\langle m\rangle)}=1+c(f-\langle f\rangle) .
$$

Here, $f$ is the differential flux, $m$ is the differential magnitude relative to a set of ensemble comparison stars, while \langle\rangle refers to mean values, given by the population means $\langle f\rangle=\int_{0}^{1} f(\varphi) \mathrm{d} \varphi$ and $\langle m\rangle=-2.5 \log \left(\int_{0}^{1} 10^{-0.4 m(\varphi)} \mathrm{d} \varphi\right)$ for phased fluxes and magnitudes, respectively. Note that by writing Eq. (1) we also assume that the two population means refer to the same state of the star, which is the reason why we defined the mean magnitude $\langle m\rangle$ with the given expression. In principle, the conversion factor $c$ can be determined from ISIS flux and daophot magnitude curves of variable stars in the outskirts of the cluster, where crowding has no effect. Equation (1) can then be applied to those variables which have only flux curves to calculate the left-hand side of Eq. (1), whose logarithm multiplied by -2.5 gives the magnitude curve with zero mean.

In Fig. 1 we plot data for two high-amplitude RR Lyrae stars which are located in the outer regions of the field. V71 is the largest-amplitude variable with no crowding, so its data were used to determine $c$. The solid dots in Fig. 1 yield a slope of $c=(-1.550 \pm 0.005) \times 10^{-5}$. Since these calibration points cover the whole range of ISIS fluxes for all the RR Lyraes, no extrapolation was needed for the other stars. For comparison, we also show data for another uncrowded star, V35. The two sets do not differ by more than $1 \%$ anywhere, so that Eq. (1), calibrated by V71 alone, can predict magnitudes from fluxes within $0.01 \mathrm{mag}$ (without the zeropoint). For a few other RR Lyrae stars we also compared the magnitude curves that were calculated from the 


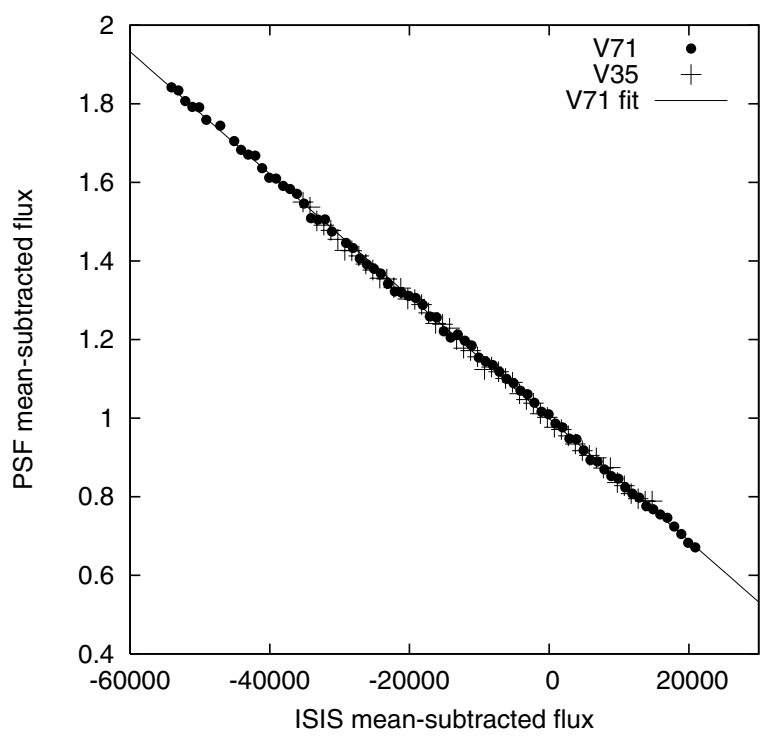

Fig. 1. The conversion between mean-subtracted flux values.

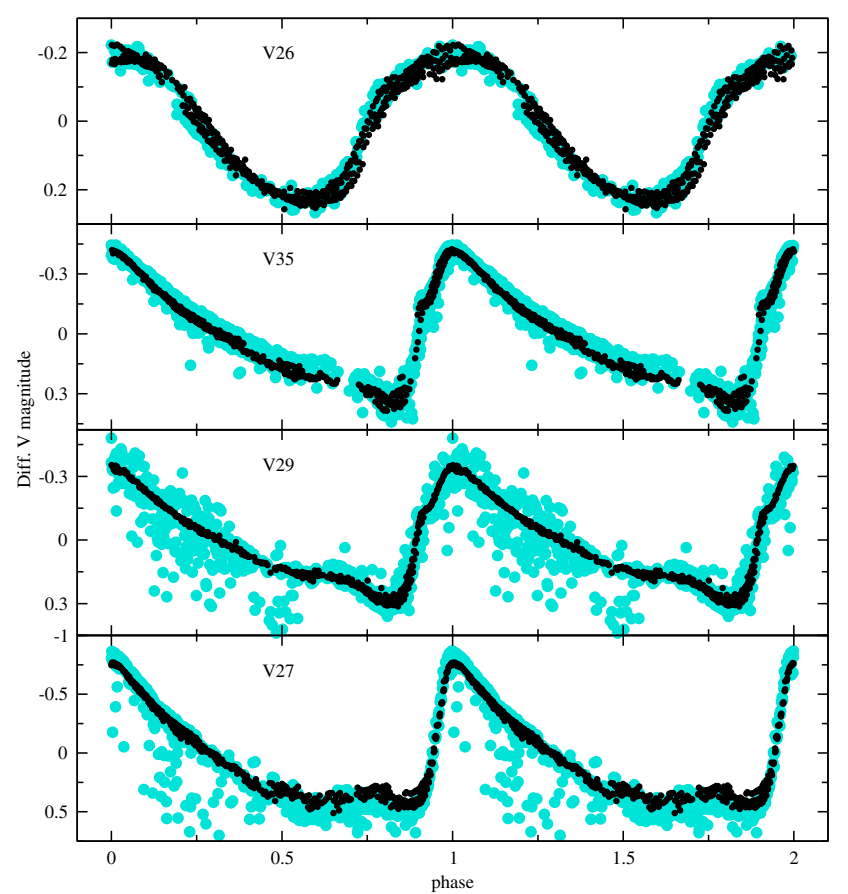

Fig. 2. Mean-subtracted magnitude curves obtained using daophot (light grey/turqoise points) and Eq. (1) applied to ISIS fluxes (black dots). The large scatter in V29 and V27 is caused by crowding.

ISIS flux data to the daophot magnitudes and always found excellent agreement (Fig. 2), thus confirming the applicability of our approach. We therefore converted ISIS flux curves to magnitudes for every star with good phase coverage.

All observations presented in this paper are available electronically at the CDS.

\section{Regular RR Lyr stars}

From the periods and light curve shapes we identified $45 \mathrm{RRab} / \mathrm{RRc}$-type variable stars. In some cases only partial phase coverage was obtained and only the RR Lyrae-class could be safely concluded. The majority have very similar mean

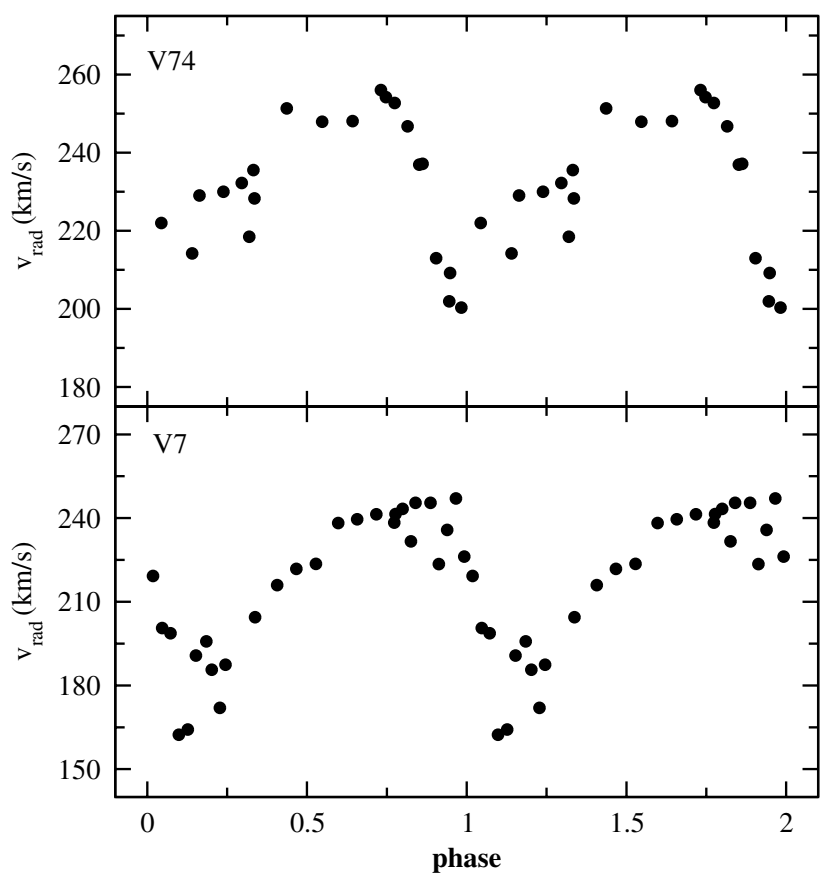

Fig. 3. Radial velocity curves for two RR Lyrae stars.

apparent brightnesses ( $V \approx 15.5 \mathrm{mag}$ ). Five of these have been observed spectroscopically (see Fig. 3 for two examples) and their mean radial velocities $\left(\approx 220 \mathrm{~km} \mathrm{~s}^{-1}\right)$ confirm membership, since the cluster's radial velocity is $223.5 \pm 2.0 \mathrm{~km} \mathrm{~s}^{-1}$ (Fischer et al. 1993). Figures 4 and 5 show sample phased/unphased data for cluster member RR Lyraes with well-defined mean light curve shapes and with partial coverage, respectively. Table 3 contains the fundamental photometric data for all the detected RR Lyrae stars. Since photometric standards were observed only on one night, $V$ brightnesses and $V-I$ colors are subject to uncertainty due to photometric variability. To address this, we calculated the pulsation phase of the standard $V$ observation of the cluster for each RR Lyrae star, so that we know from the $V$-band light curve the difference between the magnitude in that phase and the mean. The measured standard $V$ magnitudes were then corrected for these differences. With no $I$-band light curve a similar correction for the standard $I$ magnitudes was not possible, so that the $V-I$ indices may suffer from larger uncertainties (up to several tenths of a magnitude). Stars with missing $V$ and $V-I$ in Table 3 values were not detected as individual objects in the master standard frames, which means they were merged with other stars. Variables in the core of the cluster were checked for blending using an archived WFPC2 image of the Hubble Space Telescope (taken for the project "Hot stellar populations in globular cluster cores", HST Proposal 6095 by S. Djorgovski, $160 \mathrm{~s}$ exposure in the $F 439 \mathrm{~W}$ filter). In the last column of Table 3 we give comments and identifications of previously known variables from the literature.

In Fig. 6 we plotted the positions in the CMD of RR Lyrae stars with reliable calibrated photometry. Most of the stars fall on the Horizontal Branch (HB) clearly establishing membership. There are also some stars with deviant positions, for example, V80 and V81 at $V \approx 17.6$, both in the background of the cluster with distances well beyond $20 \mathrm{kpc}$. Further RR Lyraes are seen between $V=19-20 \mathrm{mag}$, which are located in the SMC.

We could determine periods, amplitudes and epochs for 28 RR Lyraes, of which 4 are first-overtone (RRc) stars. This 

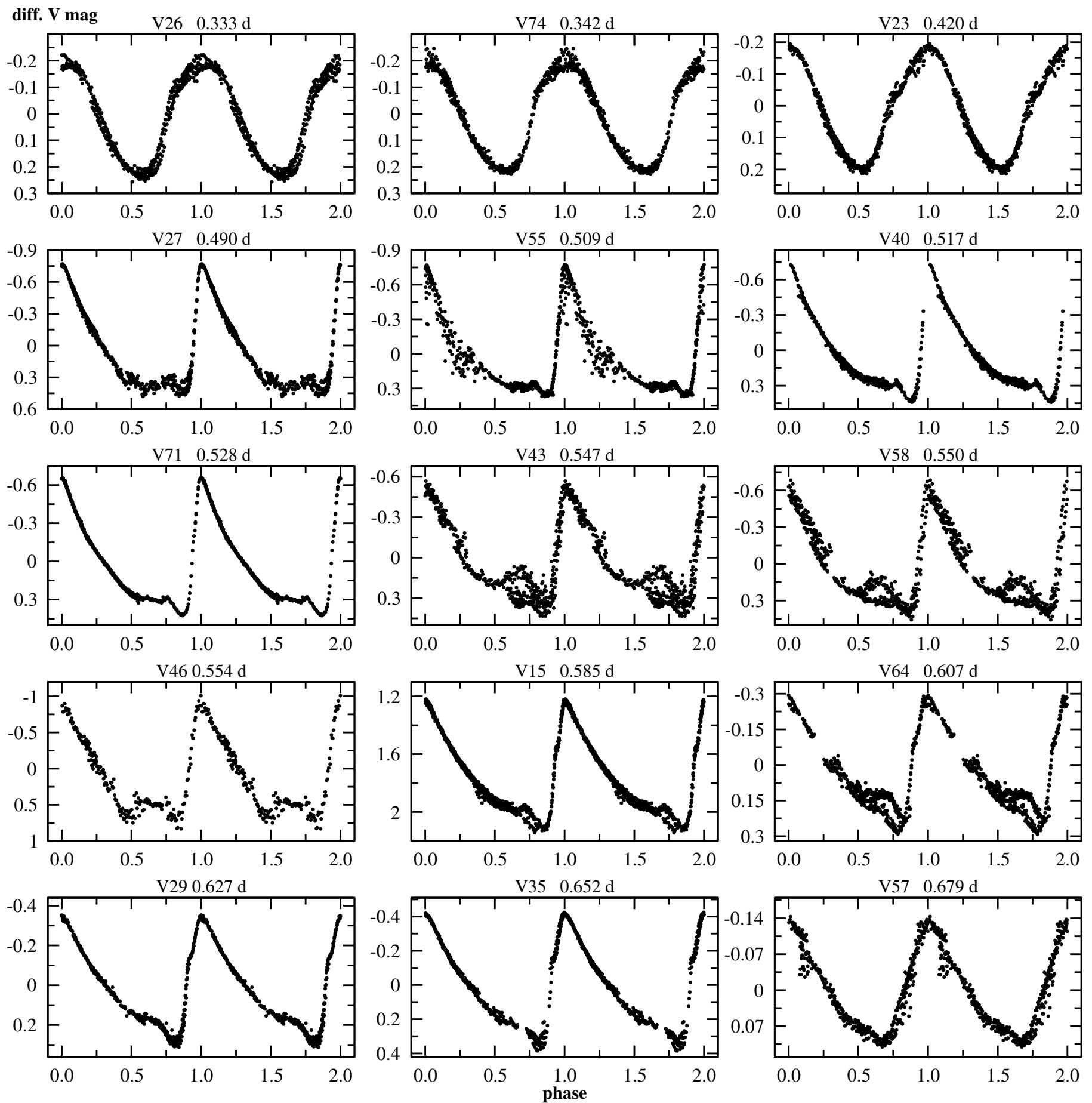

Fig. 4. Sample phased light curves of RR Lyr stars, in order of increasing pulsational period from V26 $(P=0.333 \mathrm{~d})$ to V57 $(P=0.679 \mathrm{~d})$.

fairly low percentage implies that the cluster is RRc-poor (cf. Fig. 1 of Castellani et al. 2003), which is characteristic for Oosterhoff type I globular clusters (Oosterhoff 1939). The computed specific frequency of RR Lyr variable stars, including the newly found ones, is $S_{\mathrm{RR}}=N_{\mathrm{RR}} \times 10^{0.4\left(7.5+M_{V}\right)} \approx 16$. The histogram of the periods (upper panel in Fig. 7) shows that large number of RRab stars have periods between 0.5 and $0.6 \mathrm{~d}$. The mean period of 24 RRab stars is $\left\langle P_{\mathrm{ab}}\right\rangle=0.585$ or $\left\langle\log P_{\mathrm{ab}}\right\rangle=-0.237$, while the period-amplitude relation closely follows the Oosterhoff I lines given by Clement \& Rowe (2000) and Cacciari et al. (2005). Both properties are consistent with the Oosterhoff I class (see the lower panel in Fig. 7).
In order to determine metallicities and absolute magnitudes of the RR Lyrae stars, we fitted the phase diagrams with Fourier polinomials of up to the 10th order. Using Eq. (3) of Jurcsik and Kovács (1996): $[\mathrm{Fe} / \mathrm{H}]=-5.038-5.394 P+1.345 \phi_{31}$, we estimated iron abundances for 13 RR Lyrae stars. These objects have phase curves with low scatter that covered both maxima and minima and did not show light curve modulations. We then determined absolute magnitudes using the metallicity-luminosity relation of Kovács \& Jurcsik (1996): $M_{\mathrm{V}}=0.19[\mathrm{Fe} / \mathrm{H}]+$ 1.04. Upon comparing this to our phase corrected apparent magnitudes, we calculated distance moduli for the individual stars. We determined other physical parameters (e.g. effective 

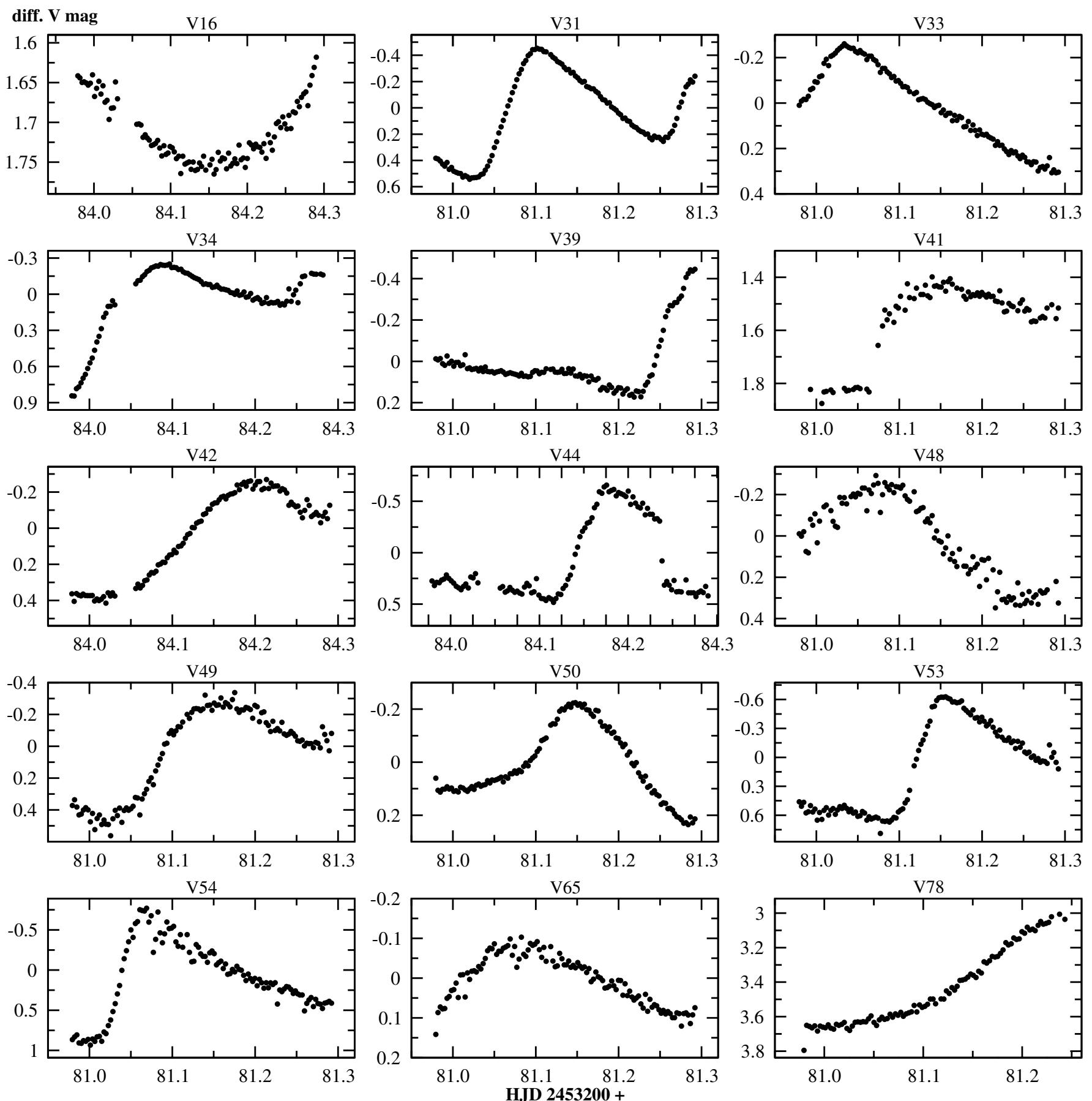

Fig. 5. Sample light curves of cluster RR Lyr stars.

temperature, luminosity) using further equations calibrated by Morgan et al. (2005), Kovács (1998) and Jurcsik (1998). The results are presented in Tables 4 and 5 .

The numbers clearly show that most of the studied RR Lyraes define reasonably small ranges of the parameters. The distance to V58 is erroneous because the star is a blend, but its other, distance-independent, parameters are similar to those of the other variables. The calculated mean metallicity of the cluster is in good agreement with values found in the literature, e.g. Caldwell (1988) and Davidge (2000), while the distance is slightly smaller, but still compatible with, the $8.5 \mathrm{kpc}$ in Harris (1996). In conclusion, RR Lyraes in NGC 362 define a consistent set of physical parameters for the cluster.

\section{RR Lyr stars with Blazhko effect}

It is known that a large fraction of RR Lyr stars (20-30\% of RRabs and $2 \%$ of RRcs, Kovács 2001) show periodic amplitude and/or phase modulations, the so-called Blazhko effect, which is one of the greatest mysteries in classical pulsating star research. In our sample, three variables, V7, V67 and V68, have strong light curve modulations, while a further 14 stars (V15, V23, V26, V27, V29, V35, V39, V40, V43, V55, V58, V64, V78, V80) exhibited subtler changes during the 15 months of observations. Furthermore, V33, V34 and V50 also showed detectable variations but these objects are in the very crowded core of the cluster and their photometry is of lower quality. 
Table 3. RR Lyrae stars in the field of NGC 362. The given coordinates are accurate within $\pm 0.1-0.2^{\prime \prime}$. Remarks on types: 1 . strong Blazhko modulation, the whole light curve is changing; 2. slight modulation in certain phases; 3. blend; 4. unsure. The last column contains other designations of already known variables: SAW xxx — Sawyer (1931); SMC Vxxx — Samus et al. (2004); MA 1315 — Meyssonnier \& Azzopardi (1993); SSP $\mathrm{xx}$ and HV $\mathrm{xx}$ - Sharpee et al. (2002).

\begin{tabular}{|c|c|c|c|c|c|c|c|c|c|}
\hline Designation & $\alpha[\mathrm{h}: \mathrm{m}: \mathrm{s}]$ & $\delta\left[{ }^{\circ}:{ }^{\prime}:{ }^{\prime \prime}\right]$ & Period [d] & $V$ & $V-I$ & $\begin{array}{l}\text { Ampl. } \\
{[\mathrm{mag}]}\end{array}$ & $\begin{array}{c}\text { Epoch } \\
{[\text { HJD-2 } 400 \text { 000] }}\end{array}$ & Type & Comments \\
\hline $\mathrm{V} 1$ & $1: 00: 56.64$ & $-71: 01: 43.8$ & 0.52835 & & & 0.55 & 2860.1765 & RRab & halo/SMC, SSP 23 \\
\hline V6 & $1: 01: 49.14$ & $-71: 07: 36.9$ & 0.30250 & 18.96 & 0.71 & 1.07 & 3284.1702 & RRab & halo/SMC \\
\hline V7 & $1: 01: 54.55$ & $-70: 46: 59.7$ & 0.54753 & 15.80 & -0.12 & 1.32 & 2854.2440 & $\mathrm{RRab}^{1}$ & SMC V1670 \\
\hline V8 & $1: 01: 56.71$ & $-71: 10: 00.2$ & 0.29218 & 19.33 & 0.83 & 0.98 & 3284.1647 & RRab & halo/SMC, SSP 29 \\
\hline V10 & $1: 01: 58.12$ & $-70: 50: 29.8$ & & & & & & RR & \\
\hline V12 & $1: 02: 00.15$ & $-70: 59: 02.3$ & & 19.77 & 1.03 & 0.35 & & RR & halo/SMC \\
\hline V15 & $1: 02: 24.02$ & $-70: 51: 56.4$ & 0.58506 & 15.55 & 0.58 & 0.91 & 2927.0715 & $\mathrm{RRab}^{2}$ & SAW V1, SMC V1712 \\
\hline V16 & $1: 02: 24.06$ & $-71: 05: 13.5$ & 0.59339 & 16.47 & 0.11 & 0.25 & 2927.1882 & $\mathrm{RRc}^{4}$ & MA 1315 \\
\hline V23 & $1: 02: 46.55$ & $-70: 51: 16.7$ & 0.42023 & 15.37 & 0.45 & 0.41 & 3281.0256 & $\mathrm{RRc}^{2}$ & SAW V10, SMC V1740 \\
\hline V26 & $1: 02: 57.22$ & $-70: 50: 40.8$ & 0.33329 & 15.07 & 1.09 & 0.47 & 3281.2486 & $\mathrm{RRc}^{2}$ & \\
\hline V27 & $1: 02: 58.08$ & $-70: 51: 23.6$ & 0.49009 & 15.50 & 0.44 & 1.44 & 3281.0826 & $\mathrm{RRab}^{2}$ & SAW V5, SMC V1572 \\
\hline V29 & 1:03:01.93 & $-70: 49: 43.2$ & 0.62709 & 15.44 & 0.58 & 0.67 & 3284.2818 & RRab & \\
\hline V31 & 1:03:04.29 & $-70: 51: 19.8$ & $\sim 0.5$ & 14.62: & 0.38: & 0.6 & & $\mathrm{RR}^{3}$ & SMC V1757 \\
\hline V33 & 1:03:05.31 & $-70: 51: 03.7$ & 0.63895 & & & 0.73 & 3281.0148 & $\mathrm{RRab}^{2}$ & \\
\hline V34 & $1: 03: 07.23$ & $-70: 50: 53.4$ & 0.63757 & 15.24 & 0.50 & 0.77 & 2927.0661 & RRab & \\
\hline V35 & $1: 03: 07.55$ & $-70: 52: 49.2$ & 0.65256 & 15.46 & 0.64 & 0.87 & 3284.1213 & $\mathrm{RRab}$ & SAW V12, SMC V1760 \\
\hline V37 & 1:03:07.88 & $-70: 50: 38.7$ & & 14.48 & 0.34 & & & $\mathrm{RR}^{3}$ & \\
\hline V38 & 1:03:07.92 & $-70: 50: 42.4$ & & 15.45 & 0.85 & & & RR & \\
\hline V39 & 1:03:08.06 & $-70: 50: 06.8$ & 0.58277 & & & 0.82 & 2855.2943 & $\mathrm{RRab}^{2}$ & \\
\hline V40 & 1:03:09.08 & $-70: 52: 00.2$ & 0.51772 & 14.96 & 0.88 & 1.17 & 3284.0559 & $\mathrm{RRab}^{2}$ & SMC V1762 \\
\hline V41 & 1:03:09.16 & $-70: 49: 45.1$ & & 15.44 & 0.81 & 0.54 & & RR & \\
\hline V42 & 1:03:09.17 & $-70: 50: 43.2$ & 0.655 & 15.43 & 0.53 & 0.69 & 3284.2027 & RRab & \\
\hline V43 & $1: 03: 11.31$ & $-70: 50: 25.4$ & 0.54714 & 15.40 & 0.93 & 1.00 & 3281.1316 & RRab & \\
\hline V44 & 1:03:11.62 & $-70: 50: 46.8$ & 0.532 & 13.60: & 1.08: & 1.14 & 3284.1753 & $\mathrm{RRab}^{3}$ & \\
\hline V45 & 1:03:11.62 & $-70: 50: 51.1$ & 0.503 & 14.03 & 0.62 & & & RRab & \\
\hline V46 & $1: 03: 12.52$ & $-70: 50: 49.8$ & 0.55417 & 12.98: & 0.63 : & 1.85 & 3281.1178 & $\mathrm{RRab}^{3}$ & \\
\hline V48 & 1:03:13.89 & $-70: 50: 53.1$ & & & & & & $\mathrm{RR}$ & \\
\hline V49 & 1:03:14.09 & $-70: 50: 41.5$ & 0.643 & & & 0.90 & 3281.1589 & RRab & \\
\hline V50 & $1: 03: 14.50$ & $-70: 51: 50.7$ & & 15.45: & 0.83: & & & $\mathrm{RR}^{3}$ & \\
\hline V53 & $1: 03: 17.19$ & $-70: 50: 43.4$ & 0.532 & & & & & $\mathrm{RRab}$ & \\
\hline V54 & $1: 03: 17.28$ & $-70: 50: 37.9$ & 0.528 & & & & & RRab & \\
\hline V55 & $1: 03: 17.57$ & $-70: 50: 15.7$ & 0.50984 & 15.49 & -0.12 & 1.15 & 3284.1132 & $\mathrm{RRab}$ & SMC V1780 \\
\hline V57 & $1: 03: 17.63$ & $-70: 49: 27.4$ & 0.67901 & 15.54 & 0.65 & 0.25 & 2929.2059 & RRab & \\
\hline V58 & 1:03:18.56 & $-70: 51: 16.7$ & 0.55060 & 13.40 & 1.33 & 1.15 & 3281.1316 & $\mathrm{RRab}^{1}$ & \\
\hline V62 & $1: 03: 21.30$ & $-70: 50: 35.2$ & & 15.56 & 0.97 & & & $\mathrm{RR}$ & \\
\hline V64 & $1: 03: 22.87$ & $-70: 50: 35.8$ & 0.60726 & 15.50 & 0.76 & 0.60 & 2929.1598 & RRab & \\
\hline V65 & $1: 03: 24.17$ & $-70: 50: 43.7$ & 0.691 & & & & & $\mathrm{RRab}^{3}$ & \\
\hline V67 & 1:03:31.24 & $-70: 50: 41.7$ & 0.51464 & 15.52 & 0.62 & 1.17 & 3281.0691 & $\mathrm{RRab}^{1}$ & SAW V6, SMC V1800 \\
\hline V68 & $1: 03: 32.57$ & $-70: 53: 19.7$ & 0.47446 & 15.60 & 1.07 & 1.10 & 3284.1810 & $\mathrm{RRab}^{1}$ & SAW V3, SMC V1804 \\
\hline V71 & $1: 03: 40.35$ & $-70: 51: 18.7$ & 0.52856 & 15.54 & 0.63 & 1.09 & 3281.0038 & RRab & SAW V7, SMC V1816 \\
\hline V73 & $1: 03: 50.37$ & $-70: 37: 51.7$ & & 19.83 & 0.81 & 0.9 & & $\mathrm{RR}$ & halo/SMC \\
\hline V74 & 1:03:56.72 & $-70: 46: 19.0$ & 0.34249 & 15.55 & 0.54 & 0.48 & 3281.2160 & $\mathrm{RRc}$ & \\
\hline V78 & 1:04:56.99 & $-70: 44: 12.2$ & 0.83238 & & & 0.72 & 2854.2549 & $\mathrm{RRab}^{2}$ & \\
\hline V80 & $1: 05: 14.82$ & $-71: 03: 10.1$ & 0.47084 & 17.66 & 0.55 & 1.28 & 3281.1234 & RRab & halo \\
\hline V81 & $1: 05: 33.24$ & $-71: 00: 43.4$ & 0.57571 & 17.71 & -0.01 & 0.76 & 2921.1516 & $\mathrm{RRab}^{2}$ & halo \\
\hline
\end{tabular}

Assuming that these light curve modulations are representative for the whole sample, the occurrence rate is rather high: about half of the cluster RR Lyrae stars show some kind of changes in the phased data over the course of our monitoring. The three stars with the strongest modulations are shown in Fig. 8, where one can see that changes in the light curve shape occurred in almost all phases. Very recently, Jurcsik and coworkers obtained high-quality observations for a number of field RR Lyraes with the Blazhko effect (Jurcsik et al. 2006; Sódor et al. 2006; Jurcsik et al. 2005), revealing many puzzling features of the observed modulations, such as the confinement of the light curve changes to a certain range of phases or the presence of doubly-periodic modulations. Our data are not extensive enough to study these phenomena, but it is obvious that there is a valuable set of RR Lyraes in the cluster. With no firm theoretical explanation of the effect, a globular cluster with many modulated RR Lyraes clearly deserves further investigations. Based on our observations, NGC 362 may be an excellent target for obtaining long-term, homogeneous photometry of variables showing the Blazhko effect.

\section{The color-magnitude diagram}

Figure 9 shows CMDs for stars within an increasing area around the cluster. The radii were chosen as $3^{\prime}, 5^{\prime}, 10^{\prime}$ and $30^{\prime}$, leading to $2450,4060,5870$ and 8567 stars in each plot. As we increase 
Table 4. Fourier and the physical parameters of selected RRab stars. Notes: ${ }^{\dagger}$ Blazhko effect present. ${ }^{x}$ Blend. ${ }^{*}$ Outliers, not included in the cluster average.

\begin{tabular}{lcccccccccccc}
\hline \hline Star & $\phi_{1}$ & $\phi_{3}$ & $\phi_{4}$ & $\mathrm{~A}_{1}$ & Period $[\mathrm{d}]$ & $(V-I)_{0}$ & $(V-K)_{0}$ & {$[\mathrm{Fe} / \mathrm{H}]$} & $\log T_{\text {eff }}$ & $\log L / L_{\odot}$ & $M_{\mathrm{V}}$ & $D[\mathrm{kpc}]$ \\
\hline V15 & 0.752 & 1.242 & 1.658 & 0.308 & 0.5851 & 0.52 & 0.59 & -1.11 & 3.867 & 1.58 & 0.80 & 8.4 \\
V27 & 0.828 & 0.917 & 1.247 & 0.494 & 0.4901 & 0.55 & 1.02 & -1.34 & 3.819 & 1.59 & 0.79 & 8.3 \\
V29 & 0.736 & 1.414 & 1.938 & 0.221 & 0.6271 & 0.51 & 0.38 & -1.04 & 3.890 & 1.58 & 0.80 & 7.9 \\
V35 & 0.805 & 1.689 & 5.513 & 0.267 & 0.6525 & 0.67 & -0.74 & -1.08 & 4.015 & 1.60 & 0.75 & 8.2 \\
V40 & 0.698 & 0.982 & 0.919 & 0.215 & 0.5177 & 0.48 & 0.67 & -0.88 & 3.858 & 1.53 & 0.93 & 6.0 \\
V43 & 0.795 & 0.992 & 1.410 & 0.299 & 0.5471 & 0.52 & 0.71 & -1.41 & 3.854 & 1.58 & 0.82 & 7.8 \\
V55 & 0.917 & 0.988 & 0.965 & 0.290 & 0.5098 & 0.54 & 0.85 & -1.71 & 3.840 & 1.58 & 0.83 & 8.2 \\
V58 ${ }^{x, *}$ & 0.822 & 1.182 & 1.239 & 0.335 & 0.5506 & 0.54 & 0.76 & -1.28 & 3.849 & 1.58 & 0.81 & 3.1 \\
V64 & 0.886 & 1.838 & -0.293 & 0.195 & 0.6072 & 0.62 & 0.78 & -0.96 & 3.845 & 1.56 & 0.84 & 8.1 \\
V67 & 0.606 & 0.780 & 0.981 & 0.339 & 0.5146 & 0.49 & 0.82 & -0.76 & 3.840 & 1.54 & 0.88 & 8.0 \\
V71 & 0.739 & 0.933 & 1.152 & 0.375 & 0.5286 & 0.53 & 0.85 & -1.16 & 3.838 & 1.57 & 0.82 & 8.2 \\
V80* & 0.904 & 1.073 & 1.450 & 0.427 & 0.4708 & 0.29 & 0.89 & -1.33 & 3.834 & 1.57 & 0.84 & 22 \\
V81 & 1.049 & 0.914 & 1.770 & 0.283 & 0.5757 & 0.40 & 0.75 & -2.69 & 3.854 & 1.66 & 0.70 & 24 \\
\hline Average & 0.78 & 1.18 & 1.52 & 0.30 & 0.557 & 0.51 & 0.60 & -1.16 & 3.865 & 1.57 & 0.82 & 7.9 \\
$\sigma$ & 0.08 & 0.32 & 1.37 & 0.08 & 0.05 & 0.08 & 0.45 & 0.25 & 0.05 & 0.14 & 0.04 & 0.6 \\
\hline
\end{tabular}

Table 5. Fourier and physical parameters of selected RRc stars.

\begin{tabular}{lccccccccccc}
\hline \hline Star & $\phi_{1}$ & $\phi_{2}$ & $\phi_{3}$ & $\mathrm{~A}_{4}$ & Period [d] & $M / M_{\odot}$ & $\log L / L_{\odot}$ & $\log T_{\text {eff }}$ & $\log Y$ & $M_{\mathrm{V}}$ & $D[\mathrm{kpc}]$ \\
\hline V23 & 0.045 & 0.498 & 1.706 & 0.0056 & 0.4202 & 1.052 & 1.93 & 3.821 & -1.042 & 0.814 & 7.7 \\
V26 & -0.160 & 1.670 & 0.313 & 0.0083 & 0.3333 & 1.134 & 1.83 & 3.839 & -0.990 & 0.816 & 6.7 \\
V74 & -0.145 & 1.233 & 0.259 & 0.0087 & 0.3425 & 1.179 & 1.88 & 3.838 & -1.001 & 0.826 & 8.3 \\
\hline Average & -0.086 & 1.133 & 0.757 & 0.0075 & 0.365 & 1.121 & 1.89 & 3.833 & -1.011 & 0.818 & 7.6 \\
$\sigma$ & 0.09 & 0.48 & 0.67 & 0.001 & 0.04 & 0.05 & 0.02 & 0.008 & 0.022 & 0.005 & 0.7 \\
\hline
\end{tabular}

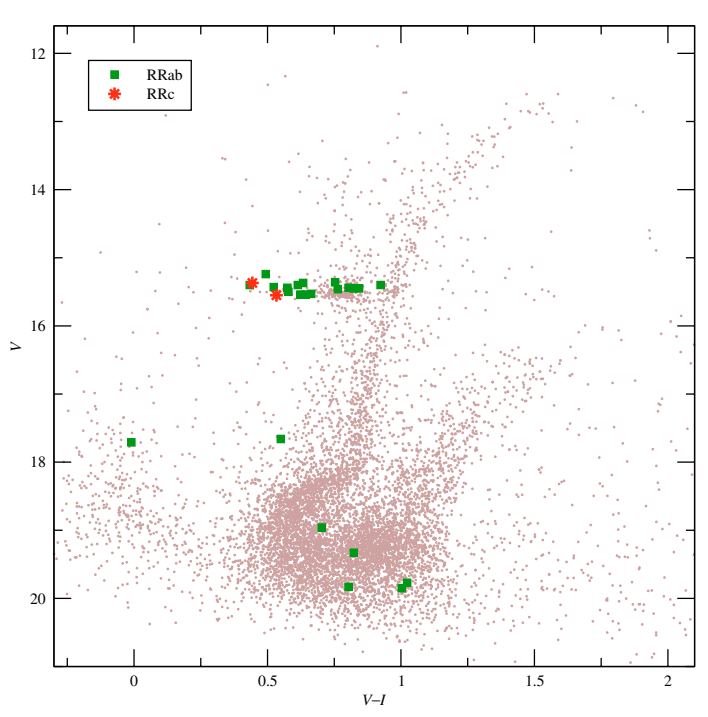

Fig. 6. RR Lyrae stars in the color-magnitude diagram of NGC 362.

the radius, the CMD exhibits an increasing field contamination and the well-defined faint secondary Red Giant Branch (RGB) and Asymptotic Giant Branch (AGB) of the SMC. We selected the second diagram (4060 stars) for isochrone fitting.

To determine the distance modulus, age, metallicity and reddening of the cluster we fitted Yonsei-Yale $\left(\mathrm{Y}^{2}\right)$ isochrones (Demarque et al. 2004). The best fit was given by the $11 \mathrm{Gyr}$ isochrone with $Z=0.001, Y=0.232,[\mathrm{Fe} / \mathrm{H}]=-1.5$ and $[\alpha / \mathrm{Fe}]=0.3$. It yields a distance modulus of $\mu=14.95 \pm 0.1$ and reddening of $E(V-I)=0.05$. The two next best fits are also shown in Fig. 10, which gave very similar distance and reddening estimates but have an age of 10 and 12 Gyr, respectively. These two fits bracket the $11 \mathrm{Gyr}$ isochrone in Fig. 10.

Between the HB and the RGB we also detect the Red Clump $(\mathrm{RC})$ as a distinct concentration of stars at $V-I \approx 0.96 \mathrm{mag}$ and $V \approx 15.5$. From the intrinsic color of the RC $(V-I=0.92$, Olsen $\&$ Salyk, 2002) and absolute brightness (for local Red Clump $M_{\mathrm{V}}=0.73$, Alves et al. 2002) we can independently estimate the reddening and distance to the cluster: $E(V-I)=0.04$ and $\mu=$ 14.82 , both in good agreement with the results of the isochrone fitting.

We corrected the distance modulus for extinction using $E(V-I) / E(B-V)=1.28$ (adopting $A_{I} / E(B-V)=1.962$ and $A_{V} / E(B-V)=3.24$, Savage \& Mathis 1979; Schlegel et al. 1998), thus $A_{I}=0.08$. In this way we arrived at $E(B-V)=0.04$ and, using $A_{I} / A_{V}=0.601$, we obtained $A_{V}=0.13$, resulting in a true distance modulus of $\mu_{0}=14.82 \pm 0.1$.

While the red HB is heavily populated, there is a noticeable lack of stars in the blue wing of the HB (see a recent discussion of this feature by Bellazzini et al. 2001 and Catelan et al. 2001). From Fig. 10 we measured the following relative age horizontal and vertical parameters (Rosenberg et al. 1999): $\delta(V-I)_{2.5}=0.305 \pm 0.05$ and $\Delta V_{\mathrm{TO}}^{\mathrm{HB}}=3.4 \pm 0.1$. Both values agree within the uncertainties with those measured by Rosenberg et al. (1999). The brightness of the horizontal branch is $V_{\mathrm{HB}}=15.53 \pm 0.08$, which is consistent with the RR Lyrbased relation $M_{\mathrm{V}}(H B)=0.22[\mathrm{Fe} / \mathrm{H}]+0.89$ relation (Gratton et al. 2003), which predicts $V_{\mathrm{HB}}=15.58$. We also calculated the Horizontal Branch morphology parameter $L$, as defined by Lee (1990): $L=(B-R) /(B+R+V)$, where $B, R$ and $V$ are the numbers of blue, red, and variable HB stars, respectively. The result is $L=-0.855$, which can be considered as an improvement to the currently available value ( -0.87 , Harris 1996), thanks to our better statistics of variable HB stars. 

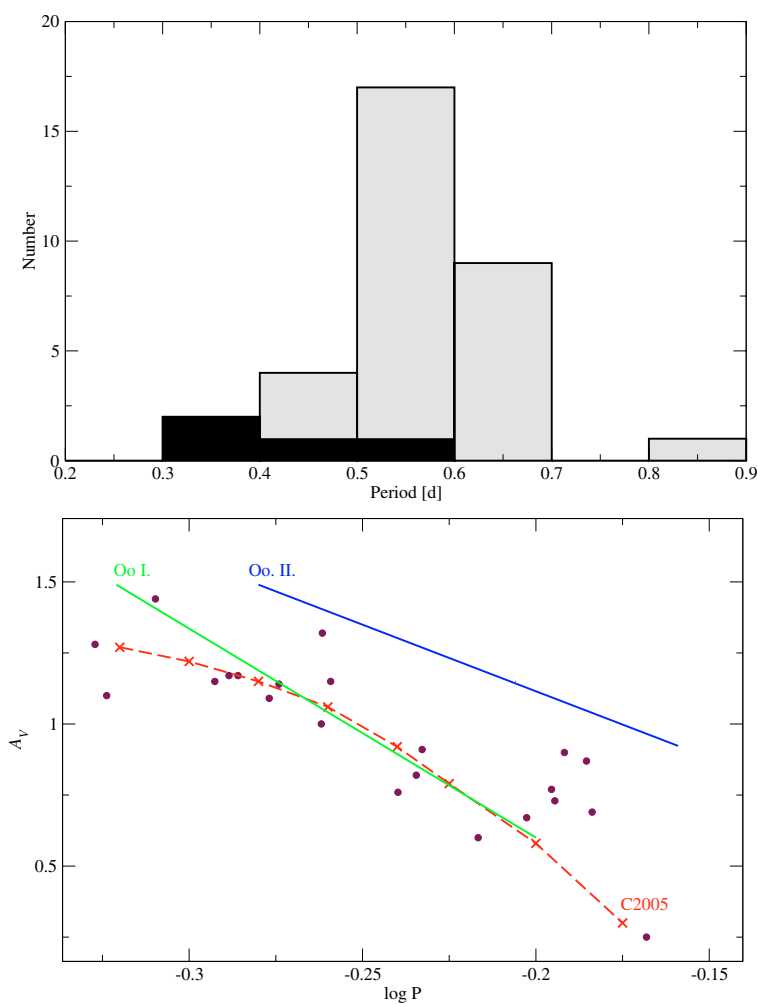

Fig. 7. Upper panel: period distribution of cluster RR Lyrae stars. The darker columns refer to the RRc stars. Lower panel: Bailey-diagram for the cluster RR Lyrae stars. Solid lines: Clement \& Rowe (2000); dashed line: Cacciari et al. (2005).

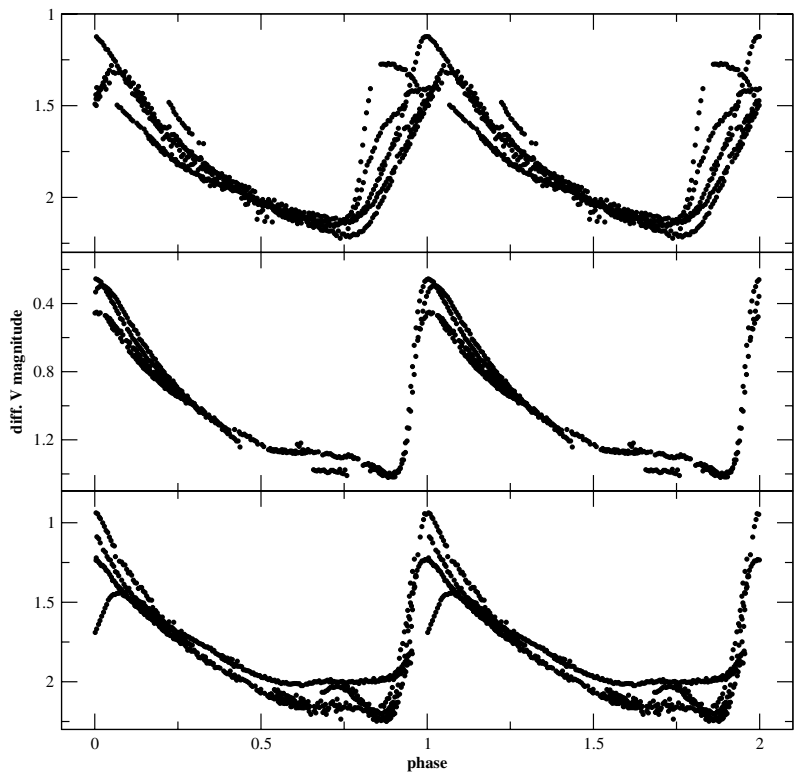

Fig. 8. RR Lyr stars with Blazhko effect. From bottom to top: V7, V67, V68.

\section{Other short-period variable stars}

We found a reasonably large number of variable stars other than RR Lyraes in the field of NGC 362. In Fig. 11 we plot the celestial positions of 8616 stars for which transformation to World Coordinate System could be achieved, with all variable stars shown separately. Apparently, most of the cluster variables are located in the central regions. The excess of variables in the lower part of the figure is caused by the Small Magellanic Cloud, located just outside of the field of view towards the south.

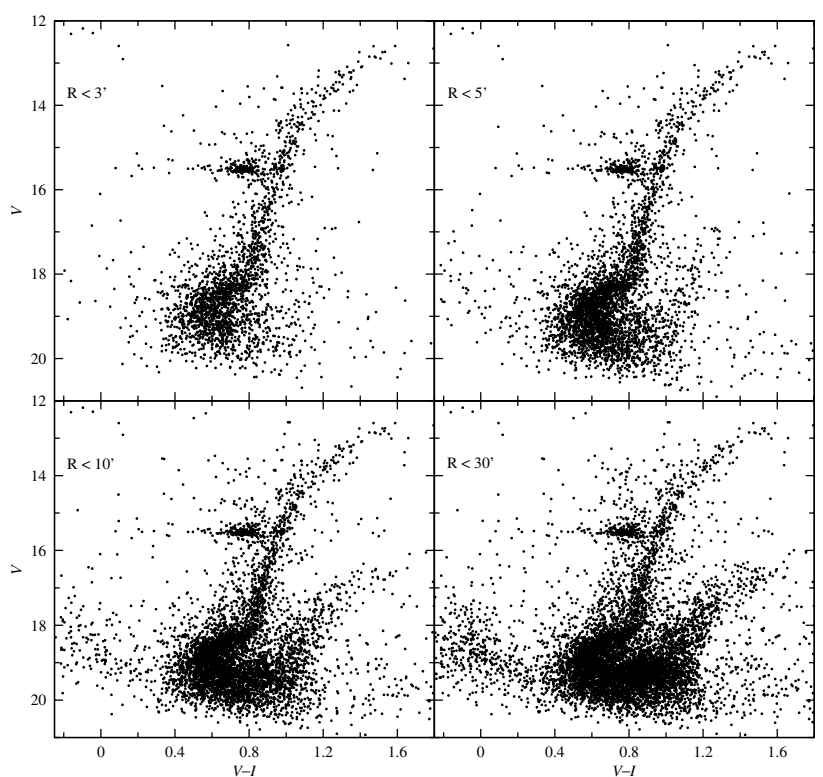

Fig. 9. $V, V-I$ CMDs with different star samples within increasing apertures centered on NGC 362. The aperture radii were 3, 5, 10 and 30 arcmin. The outer regions clearly show a secondary faint RGB/AGB caused by stars in the SMC. The isochrones were fitted to data in the upper right $\left(R<5^{\prime}\right)$ panel.

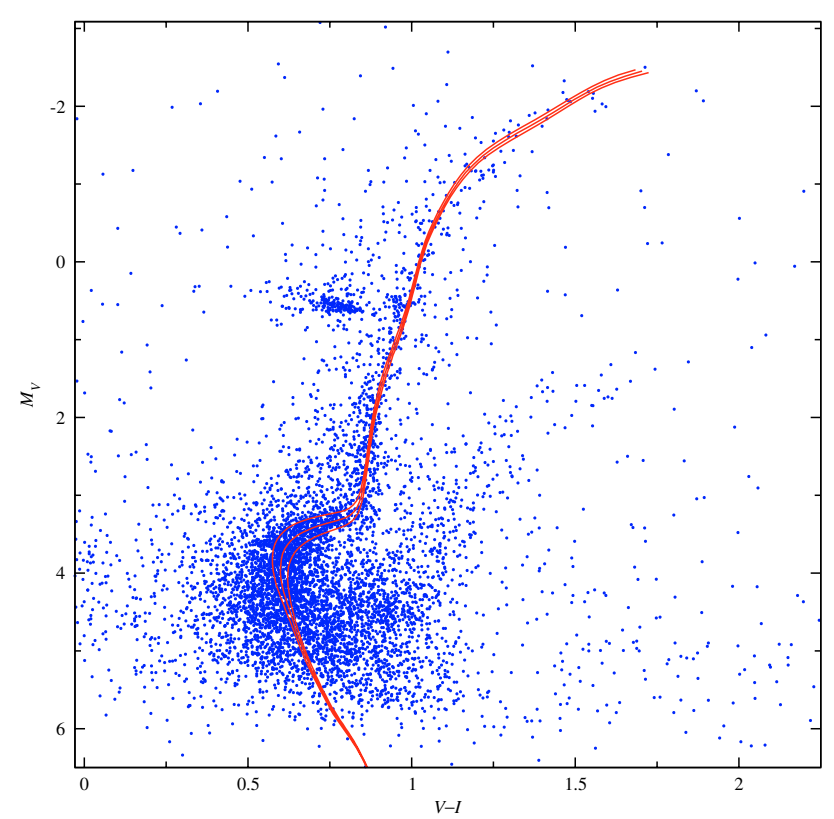

Fig. 10. $V, V-I \mathrm{CMD}$ of the cluster with fitted $Z=0.001$ isochrones for 10,11 and $12 \mathrm{Gyr}$ (from top). The derived cluster parameters are $\mu=14.82 \pm 0.10$ and $E(V-I)=0.05$.

Selected light curves are shown in Fig. 12, while the basic parameters are listed in Table 6 . Here the $V-I$ colors are again only indicative because of the single-epoch standard $I$ observation. $V$ brightnesses were corrected for variability in the same way as for the RR Lyraes. Besides eclipsing binaries, field $\delta$ Scuti stars and long-period variables, the most interesting objects include several Cepheids (three new discoveries) that can be identified as members of the Small Magellanic Cloud. Their positions in the sky fall closer to the SMC, which strengthens the identification. 
Table 6. Other variable stars in the field of NGC 362. The last column contains other designations of already known variables: SAW xxx Sawyer (1931); SMC Vxxx — Samus et al. (2004); SSP xx and HV xx — Sharpee et al. (2002).

\begin{tabular}{|c|c|c|c|c|c|c|c|c|c|}
\hline Designation & $\alpha[\mathrm{h}: \mathrm{m}: \mathrm{s}]$ & $\delta\left[{ }^{\circ}:^{\prime}:^{\prime \prime}\right]$ & Period [d] & $V$ & $V-I$ & $\begin{array}{l}\text { ampl. } \\
\text { [mag] }\end{array}$ & $\begin{array}{c}\text { Epoch } \\
\text { [HJD-2 } 400000]\end{array}$ & Type & Comments \\
\hline V2 & $1: 00: 59.28$ & $-70: 36: 33.4$ & 0.58022 & 16.58 & 0.24 & 0.42 & 2926.0898 & EA & \\
\hline V5 & $1: 01: 30.52$ & $-71: 06: 57.4$ & 2.50181 & 16.51 & 0.63 & 1.16 & 2926.9921 & Cep & \\
\hline V9 & 1:01:57.97 & $-70: 59: 28.4$ & $\sim 1.26$ & 15.65 & -0.26 & 0.38 & & EW & \\
\hline V11 & 1:01:58.90 & $-71: 04: 15.6$ & 2.01329 & 15.83 & 0.41 & 0.66 & & Cep & SMC V1684, HV 1883 \\
\hline V13 & 1:02:17.92 & $-71: 05: 21.9$ & 0.9624 & 18.49 & -0.01 & 0.58 & & EA & \\
\hline V14 & 1:02:21.92 & $-71: 10: 08.3$ & 3.0650 & 16.28 & 0.69 & 1.03 & 2923.1352 & Cep & SMC V1709, HV 1906 \\
\hline V21 & $1: 02: 42.53$ & $-71: 08: 21.6$ & 1.13503 & 16.66 & 0.50 & 0.57 & 2921.1134 & Cep & SSP 32 \\
\hline V22 & $1: 02: 45.24$ & $-71: 05: 26.2$ & 1.59262 & 16.61 & 0.47 & 0.48 & 2850.1864 & Cep & \\
\hline V28 & $1: 02: 59.45$ & $-70: 59: 16.2$ & 0.23801 & 18.94 & 1.11 & 0.70 & 3284.1647 & EW & \\
\hline V30 & 1:03:04.18 & $-71: 06: 22.8$ & 1.62998 & 17.03 & 0.59 & 1.05 & 2926.9921 & Cep & \\
\hline V47 & 1:03:13.14 & $-70: 51: 00.8$ & & & & & & EA & \\
\hline V51 & 1:03:15.19 & $-70: 50: 36.7$ & & & & & & SRA & SMC V1774 \\
\hline V52 & 1:03:17.11 & $-70: 51: 28.1$ & & 14.68 & 0.72 & 0.10 & & DS/SX & \\
\hline V56 & $1: 03: 17.63$ & $-70: 50: 49.4$ & & 12.30 & -0.16 & & & EA & \\
\hline V60 & 1:03:19.37 & $-70: 56: 03.3$ & 3.90134 & 15.86 & 0.44 & 1.12 & 2855.2316 & Cep & SAW V8 \\
\hline V63 & 1:03:22.87 & $-70: 51: 14.6$ & & 15.51 & 0.70 & 0.09 & & DS/SX & \\
\hline V69 & 1:03:34.09 & $-70: 41: 42.8$ & & 13.55 & 0.35 & 0.025 & & DS & \\
\hline V72 & $1: 03: 44.13$ & $-70: 54: 01.1$ & 1.43055 & 17.34 & 0.35 & 1.19 & 2929.1706 & Cep & SAW V15 \\
\hline V75 & 1:04:10.11 & $-70: 57: 21.5$ & 4.205 & & & 0.66 & & Cep & SMC V1851, HV 214 \\
\hline V79 & $1: 05: 15.74$ & $-70: 37: 35.7$ & 0.21630 & 19.85 & 1.01 & 0.88 & 3281.0745 & HADS & halo/SMC \\
\hline V82 & $1: 05: 38.75$ & $-70: 44: 09.7$ & 0.34999 & & & 0.40 & 2921.1326 & EW & \\
\hline
\end{tabular}

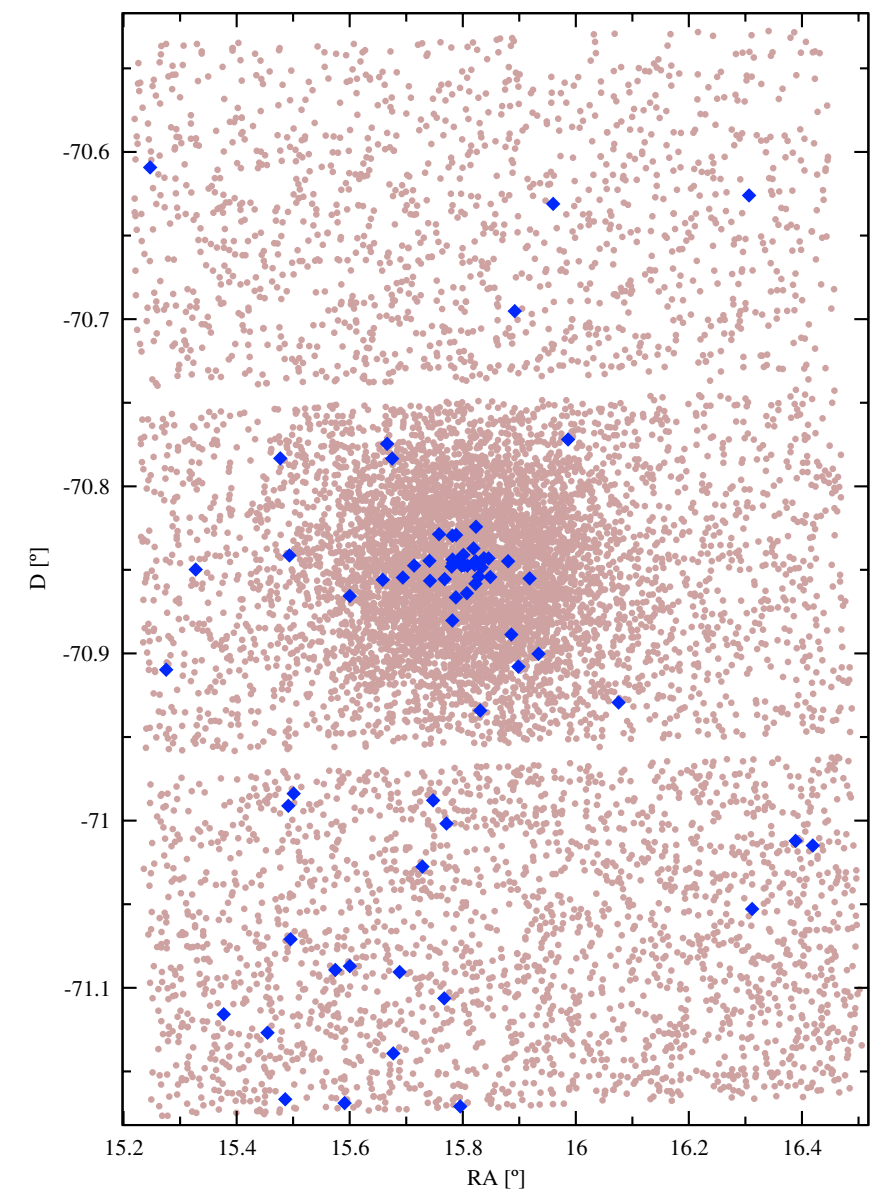

Fig. 11. RA-Dec positions of the variables stars projected on the positions of all the stars for which WCS transformation could be done. Blank sections are due to gaps between CCD segments. Note the increasing stellar density towards the SMC (in southern direction).
Table 7. Frequencies for two $\delta$ Scuti/SX Phe stars.

\begin{tabular}{lrr}
\hline \hline \multicolumn{3}{c}{ V63 } \\
\hline Frequency & $\mathrm{cd}^{-1}$ & $V$ amplitude \\
\hline$f_{1}$ & 17.1721 & 0.014 \\
$f_{2}$ & 5.26044 & 0.010 \\
$f_{3}$ & 21.4307 & 0.008 \\
$f_{4}$ & 6.00542 & 0.006 \\
$f_{5}$ & 11.8310 & 0.004 \\
$f_{6}$ & 17.8147 & 0.005 \\
\hline
\end{tabular}

$\mathrm{S} / \mathrm{N}$ for $f_{6}: 5.035$.

\begin{tabular}{lrr}
\hline \hline \multicolumn{3}{c}{ V69 } \\
\hline Frequency & $\mathrm{cd}^{-1}$ & $V$ amplitude \\
\hline$f_{1}$ & 27.6630 & 0.003 \\
$f_{2}$ & 33.0097 & 0.003 \\
$f_{3}$ & 28.7716 & 0.002 \\
$f_{4}$ & 35.8401 & 0.001 \\
\hline
\end{tabular}

$\mathrm{S} / \mathrm{N}$ for $f_{4}: 3.923$.

In Fig. 13 we plotted the locations of non-RR Lyr variables in the CMD. Blue squares show the Cepheids, which indeed occupy the expected instability strip of the SMC. Moreover, their mean $V$ magnitudes strictly follow the Cepheid periodluminosity relation $V=-2.76 \log P+17.61$, based on OGLE observations of the SMC (Udalski et al. 1999). In addition, we can clearly identify four cluster member red giant variables on the AGB and RGB and one in the SMC.

$\delta$ Sct-type variables show a large range in apparent magnitude, showing that they belong to the galactic field. Based on its colour, magnitude and proximity to NGC 362, V63 may be member of the cluster thus belonging to the SX Phe class of variable stars. Two of these stars, V63 and V69, exhibited complex 

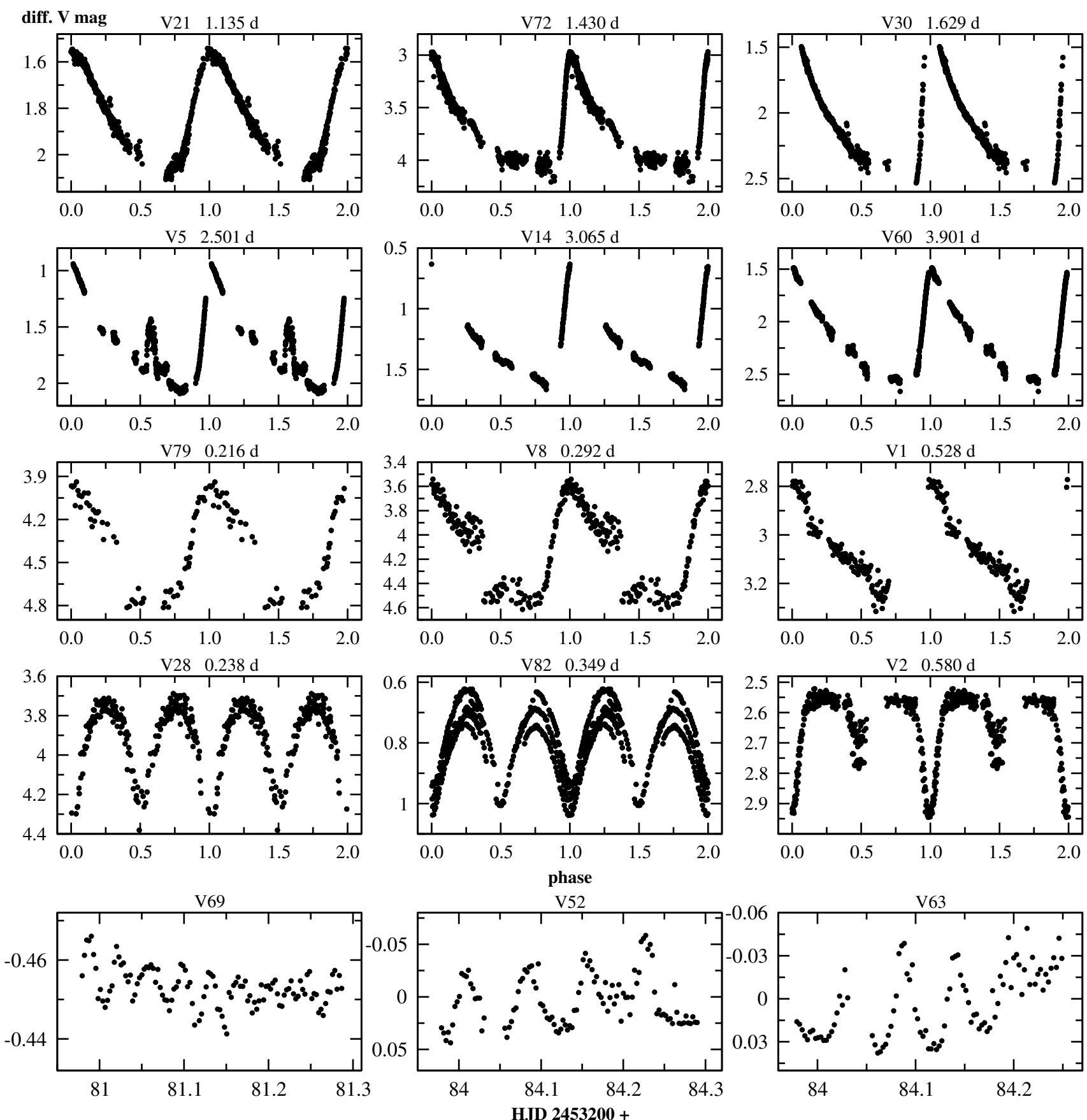

Fig. 12. Sample light curves of field variable stars. Top and second rows: Cepheids; third row: eclipsing binaries, bottom row: $\delta$ Scuti stars.

multiperiodic variations, from which we determined several pulsation frequencies with iterative sine-wave fitting using Period04 of Lenz \& Breger (2005). Although a thorough pulsation analysis of these stars is beyond the scope of this paper, we list the resulting frequencies in Table 7 . We included all frequencies with $S / N$ ratio (Breger et al. 1993) higher than 4 . For V63, we suspect that the low-frequency components can be caused by instrumental effects and/or poor sampling. Otherwise, the frequency ratios of the strongest modes suggest low-order radial pulsation, possibly flavoured with non-radial modes.

Finally, we found a number of variable stars, for which neither periods nor types could be determined. They are listed in Table 8.

\section{Conclusions}

The main results of this paper can be summarized as follows.

- Using PSF and image subtraction photometry we found 45 RR Lyr variables in the field of the cluster, of which 28 are new discoveries. We converted flux curves into magnitudes for all RR Lyrae stars with a simple method, which allowed us to use empirical light curve shape vs. physical parameter calibrations. With these, we determined metallicities, absolute magnitudes, reddenings and other physical parameters for 16 RR Lyraes.

- We found a rather high-percentage of modulated RR Lyr light curves, i.e. the Blazhko effect, both for RRab and 
Table 8. Variable stars with no period and secure classification.

\begin{tabular}{ccccccl}
\hline \hline Des. & $\alpha[\mathrm{h}: \mathrm{m}: \mathrm{s}]$ & $\delta\left[{ }^{\circ}::^{\prime \prime}\right]$ & $V$ & $V-I$ & ampl. & Notes \\
\hline V3 & $1: 01: 06.09$ & $-70: 54: 35.5$ & 16.18 & 0.28 & 0.1 & DS \\
V4 & $1: 01: 18.94$ & $-70: 51: 01.5$ & 10.98 & 1.05 & & \\
V17 & $1: 02: 37.82$ & $-70: 51: 21.8$ & 13.51 & 1.38 & & LPV \\
V18 & $1: 02: 37.93$ & $-70: 50: 42.3$ & & & & EA \\
V19 & $1: 02: 39.48$ & $-70: 46: 28.9$ & 16.83 & 1.79 & & LPV \\
V20 & $1: 02: 42.00$ & $-70: 47: 00.0$ & 16.57 & 0.30 & 0.09 & DS \\
V24 & $1: 02: 51.13$ & $-70: 50: 50.3$ & 13.94 & 1.12 & & \\
V25 & $1: 02: 54.85$ & $-71: 01: 39.3$ & 18.23 & -0.25 & 0.8 & Cep? \\
V32 & $1: 03: 05.12$ & $-71: 00: 06.3$ & 16.84 & 0.36 & 0.25 & EA \\
V36 & $1: 03: 07.84$ & $-70: 49: 46.6$ & 12.64 & 1.40 & & LPV \\
V56 & $1: 03: 17.63$ & $-70: 50: 49.4$ & 12.30 & -0.16 & & EA \\
V59 & $1: 03: 18.79$ & $-70: 50: 37.5$ & & & & \\
V61 & $1: 03: 20.35$ & $-70: 50: 54.7$ & 12.65 & 1.80 & & \\
V66 & $1: 03: 25.43$ & $-70: 50: 04.3$ & & & & \\
V70 & $1: 03: 35.11$ & $-70: 54: 29.2$ & 19.40 & 0.55 & & Cep? \\
V76 & $1: 04: 18.78$ & $-70: 55: 45.7$ & 14.76 & 1.14 & & \\
V77 & $1: 04: 26.74$ & $-70: 50: 09.8$ & & & \multirow{2}{*}{0.8} & EA \\
V83 & $1: 05: 40.59$ & $-71: 00: 53.2$ & 18.88 & 0.94 & 0.12 & LPV \\
V84 & $1: 06: 19.92$ & $-71: 04: 55.3$ & & & 0.12
\end{tabular}

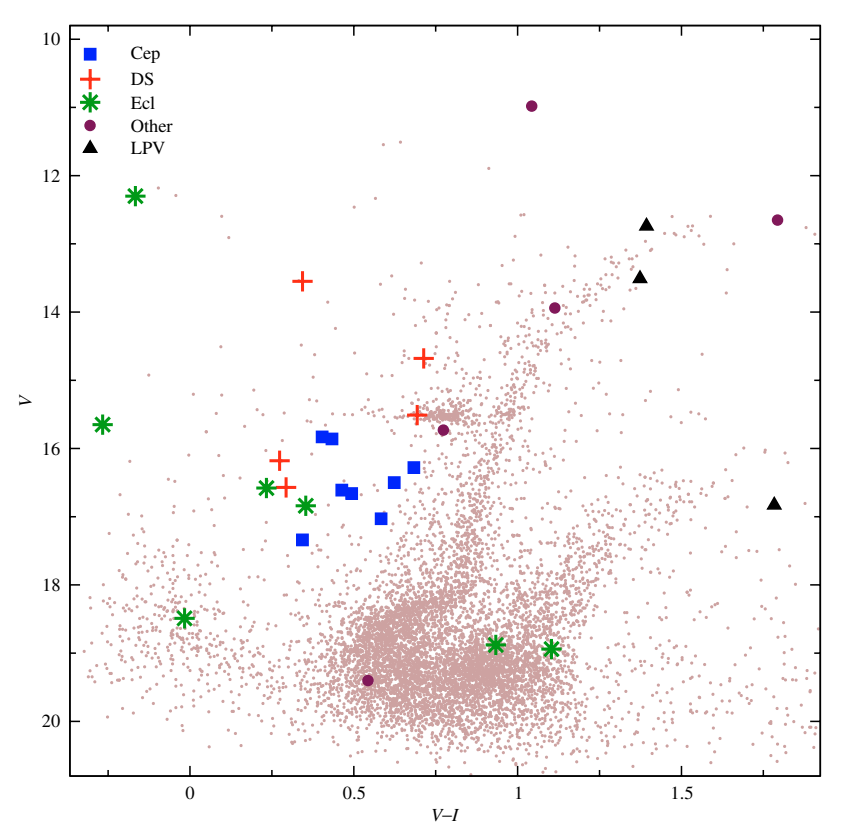

Fig. 13. Variable stars of other types than RR Lyr in the colormagnitude diagram of NGC 362.

RRc-type stars. NGC 362 could be an excellent target for studying the Blazhko effect in a chemically homogeneous environment.

- From isochrone fitting we also determined the main parameters of the cluster. Both the RR Lyraes and the colormagnitude diagram result in a consistent set of physical parameters.

- We also discovered variable stars of other types, including Cepheids and long-period variables in the Small Magellanic Cloud, eclipsing binaries and $\delta$ Sct-type pulsating stars in the galactic field.

A brief overview of the main physical parameters of NGC 362 is given in Table 9. Of these, the only significant difference is between the distance estimates using the RR Lyraes and the
Table 9. Physical parameters of NGC 362.

\begin{tabular}{|c|c|c|c|}
\hline Reference & {$[\mathrm{Fe} / \mathrm{H}]$} & Distance $[\mathrm{kpc}]$ & Age [Gyr] \\
\hline \multicolumn{4}{|l|}{ This paper: } \\
\hline RR Lyraes & $-1.16 \pm 0.25$ & $7.9 \pm 0.6$ & - \\
\hline VI CMD & & $9.2 \pm 0.4$ & $10-12$ \\
\hline \multicolumn{4}{|c|}{ Previous studies: } \\
\hline RS05 & -1.22 & - & 9.1 \\
\hline MG04 & -1.16 & _- & - \\
\hline DA05 & -1.33 & 9.3 & 8.4 \\
\hline SK00 & -1.33 & - & - \\
\hline FW93 & -1.03 & 9 & 13 \\
\hline ZW84 & -1.27 & - & - \\
\hline HR96 & -1.16 & 8.5 & - \\
\hline
\end{tabular}

RS05 - Rakos \& Schombert (2005).

MG04 - Mackey \& Gilmore (2004).

DA05 - De Angeli et al. (2005).

SK00 - Shetrone \& Keane (2000).

FW93 - Fischer et al. (1993).

ZW84 - Zinn \& West (1984).

HR96 - Harris (1996).

color-magnitude diagram. The difference is likely to originate predominantly from the RR Lyraes, whose absolute magnitudemetallicity relation is not yet settled, with a number of suggestions about nonlinear calibrations of $M_{\mathrm{V}} /[\mathrm{Fe} / \mathrm{H}]$ (see Sect. 5 of Sandage \& Tamman 2006).

Acknowledgements. This project has been supported by the Hungarian OTKA Grant \#T042509, a Hungarian Eötvös Fellowship to PSz and the Australian Research Council. L.L.K. is supported by a University of Sydney Postdoctoral Research Fellowship. The NASA ADS Abstract Service was used to access references. This work used SIMBAD and Vizier databases operated at CDSStrasbourg, France.

\section{References}

Alard, C. 2000, A\&AS, 144, 363

Alcaino, G. 1976, A\&AS, 26, 359

Alves, D. R., Rejkuba, M., Minniti, D., et al. 2002, ApJ, 573, 51

Bolte, M. J. 1986, BAAS, 18, 1037

Bolte, M. J. 1987, ApJ, 315, 469

Bellazzini, M., Pecci, F. F., Ferraro, F. R., et al. 2001, AJ, 122, 2569

Breger, M., Stich, J., Garrido, R., et al. 1993, A\&A, 271, 482

Cacciari, C., Corwin, T. M., \& Carney, B. W. 2005, AJ, 129, 267

Caldwell, S. P., \& Dickens, R. J. 1988, MNRAS, 234, 87

Castellani, M., Caputo, F., \& Castellani, V. 2003, A\&A, 410, 871

Catelan, M., Bellazzini, M., Landsman, W. B., et al. 2001, AJ, 122, 3171

Cayrel de Strobel, G., Soubiran, C., \& Ralite, N. 2001, A\&A, 373,159

Clement, C. M. 2002, Catalogue of Variable Stars in Globular Clusters http://www . astro.utoronto.ca/ cclement/read.html

Clement, C. M., \& Rowe, J. 2000, AJ, 120, 2579

Clement, C. M., Muzzin, A., Dufton, Q., et al. 2001, AJ, 122, 2587

Clementini, G., Corwin, T. M., Carney, B. W., et al. 2004, AJ, 127, 938

Corwin, T. M., Sumerel, A. N., Pritzl, B. J., et al. 2006, AJ, 132, 1014

Davidge, T. J. 2000, AJ, 120, 1853

De Angeli, F., Piotto, G., Cassisi, S., et al. 2005, AJ, 130, 116

Demarque, P., Woo, J-H, Kim, Y.-C., \& Yi, S. K. 2004, ApJS, 155, 667

Fischer, P., Welch, D. L., Mateo, M., et al. 1993, AJ, 106, 1508

Gratton, R. G., Bragaglia, A., Carretta, E., et al. 2003, A\&A, 408, 529

Harris, W. E. 1982, ApJS, 50, 573

Harris, W. E. 1996, AJ, 112, 1487

http://physwww . physics.mcmaster.ca/ harris/mwgc.dat Jurcsik, J. 1998, A\&A, 333, 571

Jurcsik, J., \& Kovács, G. 1996, A\&A, 312, 111

Jurcsik, J., Sódor, Á., Váradi, M., et al. 2005, A\&A, 430, 1049

Jurcsik, J., Szeidl, B., Sódor, Á., et al. 2006, AJ, 132, 61

Kaluzny, J., Olech, A., \& Stanek, K. Z. 2001, AJ, 121, 1533

Kovács, G. 1998, Mem. Soc. Astron. It., 69, 49 
Kovács, G. 2001, in Stellar pulsation - nonlinear studies, ed. Takeuti, M., Sasselov, D. D., Astrophys. \& Space Sci. Lib. (Dordrecht: Kluwer Academic Publishers), 257, 61

Kovács, G., Jurcsik, J. 1996, ApJ, 466, 17

Kovács, G., \& Walker, A. R. 2001, A\&A, 371, 579

Lafler, J., \& Kinman, T. D. 1965, ApJS, 11, 216

Landolt, A. U. 1992, AJ, 104, 340

Lee, Y.-W. 1990, ApJ, 363, 159

Lenz, P., \& Breger, M. 2005, CoAst, 145, 53

Mackey, A. P., \& Gilmore, G. F. 2004, MNRAS, 355, 504

Meyssonnier, N., \& Azzopardi, M. 1993, A\&AS, 102, 451

Monella R. 1985, Coelum LIII, 287

Morgan, D. H., \& Hatzidimitriou, D. 1995, A\&AS, 113, 539

Morgan, S. M., Wahl, J. N., \& Wieckhorst, R. M. 2005, [arXiv: astro-ph/0506348]

Olsen, K. A. G., \& Salyk, C. 2002, AJ, 124, 2045
Oosterhoff, P. Th. 1939, Observatory, 62, 104

Rakoos, K., \& Schombert, J. 2005, PASP, 117, 245

Rosenberg, A., Saviane, I., Piotto, G., \& Aparacio, A. 1999, AJ, 118, 2306

Samus, N. N., Durlevich, O. V., et al. 2004, Combined General Catalogue of Variable Stars, VizieR On-line Data Catalog: II/250

Sandage, A., Tamman, G. A. 2006, ARA\&A, 44, 93

Savage, B., \& Mathis, J. 1979, ARA\&A, 17, 73

Sawyer, H. B. 1931, Harvard College Observatory Circular, 366, 1

Schlegel, D. J., Finkbeiner, D. P., \& Davis, M. 1998, ApJ, 500, 525

Sharpee, B., Stark, M., Pritzl, B., et al. 2002, AJ, 123, 321

Shetrone, M. D., \& Keane, M. J. 2000, AJ, 119, 840

Sódor, Á., Vida, K., Jurcsik, J., et al. 2006, IBVS, No. 5705

Stellingwerf, R. F. 1978, ApJ, 224, 953

Udalski, A., Soszynski, I., Szymanski, M., et al. 1999, AcA, 49, 437

Walter, K. 1983, A\&A, 128, 391

Zinn, R., West, M. J. 1984, ApJS, 55, 45 
P. Székely et al.: RR Lyrae stars in the southern globular cluster NGC 362, Online Material p 1

\section{Online Material}




\section{Notes on some individual stars}

In this section we give additional information for some of the detected variable stars.

V1: The given period in Sharpee et al. (2002, hereafter S02) is $1.16736 \mathrm{~d}$, however, their phase diagram shows quite large scatter. Since our data define a much smoother phase curve, we prefer the period value listed in Table $3(0.52835 \mathrm{~d})$.

V4: ISIS revealed the small-amplitude variations of this object only on one night.

V5: This Cepheid variable had a peculiar bump on the descending branch of its light curve. This phenomenon was detected only one night, possibly caused by instrumental effects.

V7: Based on our spectroscopic observations the mean radial velocity of this star is $210 \mathrm{~km} \mathrm{~s}^{-1}$.

V8: The period in S02 is 0.57382 d, however their light curve is rather noisy. With full phase coverage our period $(0.29218 \mathrm{~d})$ seems to be the correct one.

V10: High-amplitude, continouos variation with a descending branch of the flux curve, characteristic of an RR Lyr star.

V11: We confirm the period and classification by S02.

V12: We have only one night of data; the star is far from the cluster and is 4 magnitude fainter than the HB - likely in the SMC.

V14: We confirm the period and classification by S02.

V17: The star lies on the RGB of the cluster. The phased flux curve suggests a period of $69 \mathrm{~d}$.

V19: This star is located at the tip of the RGB of the SMC, possibly a long period red giant variable. The object is listed in the catalogue of carbon stars in the SMC compiled by Morgan \& Hatzidimitriou (1995).

V21: We confirm the period and classification by S02.

V23: Clement (2002) catalogued the object as a fundamental mode Cepheid with $4.20519 \mathrm{~d}$ period. That is an error due to a mistyped decimal point, because the star is an RR Lyrae variable with $0.420234 \mathrm{~d}$ period (ten times smaller). Its mean radial velocity is $230 \mathrm{~km} \mathrm{~s}^{-1}$.

V31: The star showed characteristic RR Lyr-like light curves but also a strong modulation from day to day. Both the extrema and the amplitude varied on a short time-scale, thus our efforts to determine the pulsational parameters were not successful. After checking an archived HST image, we found the star to be a very close blend. The constituting stars are separated only by 159 mas from each other and seem to have the same brightness. Therefore, we concluded that both components are RR Lyrae stars and the superposition of their individual light curves caused the failure of the period determination. Their angular separation corresponds to $1200 \mathrm{AU}$ at the distance of the cluster, so they may be physically related objects.

V36: It is included in the Catalogue of $[\mathrm{Fe} / \mathrm{H}]$ values for $\mathrm{F}, \mathrm{G}$, $\mathrm{K}$ stars (Cayrel de Strobel et al. 2001) with $[\mathrm{Fe} / \mathrm{H}]=-1.33$, in accordance with the metallicity of the cluster.

V38: This star is on the HB, while one night of ISIS data showed an ascending branch of the flux curve.

V41: This star is on the HB with an amplitude larger than $0.5 \mathrm{mag}$.

V47: This star is listed in the catalog of Galactic Globular Clusters (Monella 1985) with the following parameters: $[\mathrm{Fe} / \mathrm{H}]=-1.39, v_{\text {rad }}=-221 \mathrm{~km} \mathrm{~s}^{-1}$, clearly belonging to the cluster. The light curve is characteristic of an Algol-type eclipsing binary with hints of small amplitude pulsations in maximum. Unfortunately, it is located in a very crowded field so that the light curve is often noisy.

V48: The one night of ISIS data shows high-amplitude sinusoidal light variation. The star is located in the center of the cluster and is possibly an RRc star.

V50: The phased ISIS flux data show strong light curve variations; problems with crowding in the cluster center.

V51: Samus et al. (2004) gives a period of $135 \mathrm{~d}$.

V60: We confirm the results by Sawyer (1931).

V61: This object is located at the tip of the RGB of the cluster, probably a long-period red giant variable.

V62: The star is on the HB, suggesting that it is an RR Lyr variable. The short flux curve shows an ascending branch.

V63: Although the star seems to be on the HB, its light curve is more characteristic of a $\delta$ Sct-type variable.

V74: The mean radial velocity of the star is $230 \mathrm{~km} \mathrm{~s}^{-1}$.

V75: This star was in the gap between the CCD segments at the time of standard observation, that is why there are no $V$ and $V-I$ values in Table 6 . The light curve confirms the period and classification by $\mathrm{S} 02$.

V82: This W UMa-type variable has interesting changes in the mean level of the light curve. We could not find any source of systematical errors in our data reductions, so the behaviour seems to be real (see also Walter 1983 discussing the so-called Kwee-effect) 